\title{
The frequency distribution of presenting symptoms in children aged six months to six years to primary care
}

\author{
Sara Whitburn ${ }^{1}$, Céire Costelloe $^{2}$, Alan A. Montgomery ${ }^{3}$, Niamh M. Redmond $^{4}$, Margaret Fletcher ${ }^{5}$, \\ Tim J. Peters ${ }^{6}$ and Alastair D. Hay \\ ${ }^{1}$ Honorary GP Research Fellow, Academic Unit of Primary Health Care, NIHR School for Primary Care Research, \\ Department of Community Based Medicine, University of Bristol, Bristol, UK \\ ${ }^{2}$ Research Associate, Academic Unit of Primary Health Care, NIHR School for Primary Care Research, \\ Department of Community Based Medicine, University of Bristol, Bristol, UK \\ ${ }^{3}$ Reader in Primary Care Research, Academic Unit of Primary Health Care, NIHR School for Primary Care Research, \\ Department of Community Based Medicine, University of Bristol, Bristol, UK \\ ${ }^{4}$ Trial Coordinator, Academic Unit of Primary Health Care, NIHR School for Primary Care Research, \\ Department of Community Based Medicine, University of Bristol, Bristol, UK \\ ${ }^{5}$ Reader in Children's Nursing, Faculty of Health and Social Care, University of West England, \\ Coldharbour Lane, Bristol, UK \\ ${ }^{6}$ Professor of Primary Care Health Services Research, Academic Unit of Primary Health Care, NIHR School for \\ Primary Care Research, Department of Community Based Medicine, University of Bristol, Bristol, UK \\ ${ }^{7}$ Consultant Senior Lecturer in Primary Health Care, Academic Unit of Primary Health Care, NIHR School for Primary \\ Care Research, Department of Community Based Medicine, University of Bristol, Bristol, UK
}

Background: Primary care providers and researchers wishing to estimate study recruitment rates need estimates of illness frequency in primary care. Previous studies of children's symptoms have found that presentations are most common for the symptoms: cough, fever, earache, rash, diarrhoea and vomiting. Since 2000, primary care provision in the United Kingdom has changed with the introduction of Walk-inCentres (WICs) and new Out of Hours (OoHs) providers. Aims: To describe the type and frequency of parent-reported presenting symptoms at a range of primary care sites between 2005 and 2007. Methods: Parent-reported presenting symptoms, recorded in their own words, were extracted from data collected from all children aged six months to six years during recruitment to a randomised controlled trial. Presenting symptoms were coded and presented as frequency per 100 'consulting sessions' by type of primary care site. Findings: Results were evaluated from 2491 episodes of illness at 35 sites. When grouped by primary care site, respiratory symptoms were the most common at OoHs centres, the WIC and general practitioner (GP) surgeries. Trauma symptoms were common in the Emergency Department, but unexpectedly, diarrhoea and vomiting were more common in the Emergency Department and skin presenting symptoms more common at the WIC than at GP sites. Conclusions: We report the relative frequency of acute symptoms by type of primary care provider. These data may be useful to those planning recruitment to primary care paediatric studies and policy makers for planning primary care service provision.

Key words: child; epidemiology; illness behaviour; primary health care

Received 7 December 2009; accepted 16 September 2010; first published online 13 October 2010

\footnotetext{
Correspondence to: Dr Sara Whitburn, Rowville Health, 12-14 St Lawrance Way, Rowville VIC 3178, Australia. Email: SWhitburn@rowvillehealth.com
}

(C) Cambridge University Press 2010 


\section{Background}

Preschool children have frequent episodes of illness leading to more primary care consultations than any other age group (McCormick et al., 1995; Rowlands and Moser, 2002). However, only around half of illnesses are brought to the attention of primary care (Pattison et al., 1982). Previous studies have found that factors associated with parents' decision to consult are socio-economic, (Little et al., 2001) parental illness attitudes (Osman and Dunt, 1995) and concerns about illness severity (Wyke et al., 1990; Kai, 1996).

Several of these studies have used diagnoses from general practitioners (GPs) and general practice databases (Rowlands and Moser, 2002) or diaries that used symptoms determined by the researchers (Pattison et al., 1982; Wyke et al., 1990; Osman and Dunt, 1995). However, the use of diagnostic labels between practitioners can be inconsistent (Stocks and Fahey, 2002). This has led to calls for the research of acute problems in primary care to be based on presenting symptoms rather than disease categories (Medical Research Council, 1997).

Studies that have examined the frequency of symptoms include one conducted 30 years ago (Morrell, 1970) and three with data collection that occurred in the 1990s (Holme, 1995; Bruijnzeels et al., 1998; Hay et al., 2005). These studies found that the most common presenting symptoms of children and adolescents were cough, fever, earache, rash and gastrointestinal disturbances. Since 2000 , the structure of UK primary care service delivery has changed, with the advent of Walkin-Centres (WICs) and changes to the ways in which GP Out of Hours (OoHs) services are delivered (Department of Health, 2000). Emergency Departments also provide an open access service, with up to $40 \%$ of attendees requiring primary care treatment (Robertson-Steel, 1998). To our knowledge, no study has described the presenting symptoms of preschool children whose parents have chosen to present to these services. This information is of particular relevance to researchers wishing to estimate study recruitment rates. Many studies fail to recruit to target, often because of inaccurate estimations of the frequencies with which eligible participants present. The frequency and type of presenting symptoms would also be of interest to primary care providers to assist with planning for workforce deployment, development and training that depends on parents' health-seeking behaviour.

Therefore, the aim of this study was to describe the type and frequency of presenting symptoms in preschool children attending a range of primary care sites between 2005 and 2007.

\section{Methods}

\section{Recruitment}

Parent-reported presenting symptoms were recorded during recruitment to the Paracetamol plus Ibuprofen for the Treatment of fever in Children (PITCH) study (Hay et al., 2008). The data were collected as part of the recruitment phase of the PITCH study.

PITCH was a single centre (multi-site), individually randomised, blinded, three-arm trial investigating the relative clinical and cost effectiveness of different antipyretic strategies in children between six months and six years who could be managed in the community. Recruitment was conducted within a 12-mile radius of Bristol covering a wide variety of socio-economic dwellings, commenced in January 2005 and completed in May 2007. All Bristol National Health Service (NHS) primary care organisations, including General Practices, three OoHs General Practice Cooperatives, an NHS WIC and the Bristol Royal Children's Hospital Emergency Department were invited to support recruitment.

Three methods were used to recruit children to the PITCH study, known as 'local', 'remote' and 'community'. These have been described in detail elsewhere, (Hay et al., 2008) but in brief, the 'local' recruitment method consisted of a research nurse being stationed in the waiting rooms of collaborating NHS sites during consulting sessions (eg, mornings, afternoons or evenings). These lasted approximately 4-5 hours. Each 'session' was given a unique identifying number and only one nurse was present at each site per session. The research nurse would approach parents or carers of any child appearing to be in the appropriate age range and give them a trial 'Invitation Letter' and 'Summary Parent Information Sheet', printed on site headed paper. Receptionists were also asked to give the trial paperwork to appropriate parents. 
This initial meeting was to assess the eligibility of children approached to be enrolled in the PITCH trial. Parents were asked to indicate, if their child was in the eligible age group, and whether the child had any 'fever indicators' as the study was a trial of antipyretics. These 'indicators' were the presence of current or recent $(<24$ hours) fever. Parents responding positively to both questions were invited to read the trial paperwork and to discuss the study further with the research nurse. Parents who did not want to be involved in the trial at this stage were asked to indicate their reasons and no further data were collected. Parents responding negatively to the fever indicator questions were asked 'What was your reason for attendance at the doctor today?' The research nurse would then note the parent or carer's answers verbatim on the trial paperwork. The data were stored and analysed retrospectively. Symptom data were collected for all presentations and symptoms in children re-presenting at another session were recorded as new 'symptom episodes'. The research nurses made every effort to talk to the parents of all potentially eligible children entering the site waiting room during each session. The number for whom this was not possible was recorded.

Parents willing to proceed with the trial would then see a clinician and clinical data were collected for the PITCH study. These and further data collected during the PITCH study were not used as they did not include any further parent-reported presenting symptoms. The remote and community recruitment methods were not used to provide data for this study, as they did not allow for documentation of children's presenting symptoms.

\section{Data collection management and analysis}

A child's episode was excluded if the child was not aged between six months and six years, if the parent's/carer's English was inadequate to complete the questions or the parent/carer declined to participate. Children's illness episodes were also excluded if the research nurse logged the child's presence and a data sheet number was recorded, but the nurse did not have the opportunity to discuss the study questions with the child's parent/carer.

A Microsoft Access database was used to enter data collected from the recruitment invitation letter depending on the answer to the 'fever indicator' question. When the answer was 'no', then the parent-reported reason for attendance was entered in words or phrases verbatim by the research nurse as the presenting symptom. Each time a new presenting symptom was identified, a new code was given using the research nurses' notated parent-reported words and phrases as closely as possible. If the parent-reported reason for an attendance was not a symptom, the description of reason was initially coded like a presenting symptom to allow analysis. These were subsequently excluded at final data analysis, as our aim was to focus on parent-reported presenting symptoms. If no word or phrase that could be coded as a presenting symptom was given, this was coded as "no fever - no other presenting symptom' and was regarded as ineligible data. If the answer to the 'fever indicator' question was 'yes', then the invitation letter was scanned for details of another parent-reported presenting symptom. If none was found, this was coded 'fever no other presenting symptom' and was regarded as ineligible for the study. This data were regarded as ineligible since the first question asked by the research nurse about the presence of fever indicators in the last 24 hours was a closed question, and not a true representation of a parent-reported symptoms or reason for the consultation.

If the research nurse had recorded the word 'fever' as the parent-reported reason for attendance, then this was considered to be a presenting symptom and was recorded in the database. If more than one symptom was given, then both symptoms were coded as 'dual symptoms'. The 'dual symptoms' were then reviewed to see if the two symptoms could be grouped together and given their own presenting symptom code, for example, 'cough and cold' or 'cough and earache'.

\section{Descriptive statistics}

Place of presentation was grouped into four site types: the Emergency Department, WIC, OoHs Cooperatives, and the GP surgeries. We used frequencies (percentages) to describe parentreported presenting symptoms by symptom type (eg, respiratory) overall and then by site of presentation (eg, GP practice). The frequency of parent-reported presenting symptom was also calculated for the day/date of presentation and per 100 research nurse sessions. The frequency of 
eligible symptoms per session for one session was also calculated for each primary care site.

In order to assess the intensity of recruitment at each type of site, the median and interquatile range of research nurse sessions per month at each site was calculated. The mean of eligible number of presentation at each site was also used to assess the success of data collection at each site.

The date of each session was used to assign a week number to that session to allow analysis by calendar month.

\section{Findings}

\section{Participants}

A total of 35 sites agreed to take part in local recruitment, consisting of one WIC, the Children's Emergency Department, two GP OoHs Cooperatives and 30 general practices. Figure 1 shows the flow of children's illness episode presentations through the study. During local recruitment, research nurses approached children and their carers 3746 times. Of these, 117 presentations

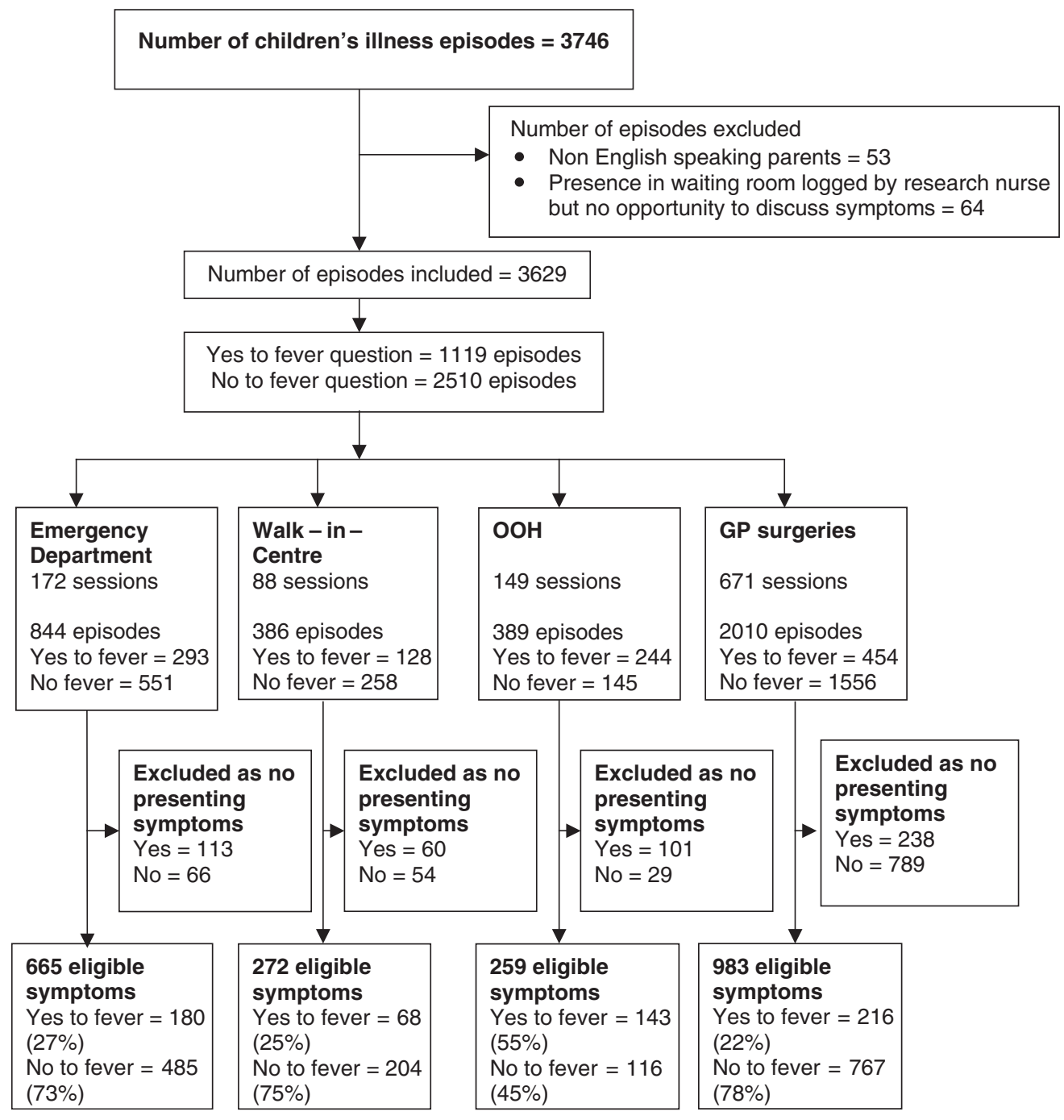

Figure 1 Flow diagram of children's illness episodes presentations

Primary Health Care Research \& Development 2011; 12: 123-134 
were excluded due to the carer's insufficient knowledge of English (53), insufficient time because the child was called to see the clinician (33), or because the parent declined to participate (31). All the children were between the ages of six months and six years. No further demographic data for our study is available as further demographic data was only collected if the child was recruited to the PITCH study. Of the remaining 3629 presentations 1119 parents answered yes to the 'fever indicator' question, of which 607 (54\%) presentations had another (eligible) parent-reported symptom or reason description. For the 2510 episodes, where parents responded no to the 'fever indicator' question there were 1884 (75\%) episodes that had a parent-reported presenting symptom or reason description. Overall, parent-reported symptom/ reason description data were present for 2491 $(69 \%)$ of children's illness episodes. There were 312 children illness episodes whose reason for attendance was not a parent-reported presenting symptom but descriptions of follow-up or routine medical care. Once these were excluded, there were 2179 eligible children illness episodes.

\section{Site of presentation}

There were 1080 recruiting sessions across the 35 participating primary care sites. Figure 1 also shows the flow of children's presentations across the four types of site. The median (quartile 1, quartile 3 and interquartile range) for the number of research nurse sessions completed per month per site were $5(3,9,6)$ for the Emergency Department; $3(2,5,3)$ for the WIC; $5(3,7,4)$ for the OoHs services and $20(16,28,12)$ for the GP surgeries. The frequency of number of eligible symptoms recorded by the research nurse in one session by site was 3.9, 3.1, 1.7 and 1.9 for the Emergency Department, WIC, OoHs services and GP surgeries, respectively.

\section{Parent-reported presenting symptoms}

Table 1 shows the type and frequency of parentreported presenting symptoms. The most frequent parent-reported symptoms grouped by type were respiratory symptoms $(30 \%)$, followed by trauma problems $(20 \%)$, skin symptoms $(18 \%)$, gastroenterological symptoms (11\%), non-specific symptoms $(9 \%)$, eye symptoms $(6 \%)$, genitourinary problems $(2 \%)$, fits $(1 \%)$ and 'other' problems $(3 \%)$. When parents reported a gastroenterological problem, they most often described the presenting symptom as vomiting and/or diarrhoea (84\%). Overall, 59\% of skin-presenting symptoms were described by parents as a 'rash'.

\section{Parent-reported presenting symptoms by site of presentation}

Table 2 shows the type and frequency of parentreported presenting symptom per site respiratory presenting symptoms were similar across sites and were the most frequent at all sites except the Emergency Department, where trauma was the most frequent presentation followed by respiratory and then gastroenterological symptoms. For the WIC the most frequent parent-reported presenting symptoms were equally spread between respiratory symptoms, trauma and skin symptoms. The frequency of parent-reported skin symptoms presenting to the WIC was twice that of those seen in OoHs and GP services. For OoHs sites, the most frequent was respiratory symptoms followed by gastroenterological and skin. At the GP surgery, the most frequent was respiratory symptoms followed by skin problems.

\section{Parent-reported presenting symptoms over time}

Figures 2-5 show the frequency of parentreported presenting symptoms per session during each month of the two-year study period grouped by type. As expected, frequency of respiratory symptoms increased during the winter months and decreased during the summer months. There was no obvious seasonal pattern for the frequency of trauma parent-reported presenting symptoms. The frequency of gastroenterological symptoms peaked in the March of both 2005 and 2006. Skin parent-reported presenting symptom frequencies increased during March to May and decreased during winter months. There was an observed decline in the frequency of parent-reported presenting symptoms over the time for all types of parent-reported presenting symptoms.

\section{Discussion}

\section{Summary of main findings}

This study shows that the most common parentreported presenting symptoms, across a broad 
Table 1 Number of presenting symptoms, percentage of all symptoms and rates per 100 sessions

\begin{tabular}{|c|c|c|c|}
\hline Presenting symptom & $n$ & $\begin{array}{l}\text { Percentage of symptoms } \\
(\% \text { of all symptoms } \\
n / 2179 \times 100)\end{array}$ & $\begin{array}{l}\text { Frequency }(n / 1080 \times 100 \text {; } \\
\text { Total } 1080 \text { sessions) }\end{array}$ \\
\hline Respiratory & 659 & 30 & 61 \\
\hline Cough & 178 & 8 & 16 \\
\hline Earache/ear infection & 129 & 6 & 12 \\
\hline $\begin{array}{l}\text { Dual respiratory - cough and another presenting } \\
\text { symptom }\end{array}$ & 65 & 3 & 6 \\
\hline Wheeze & 54 & 2 & 5 \\
\hline Orthopaedics and trauma & 433 & 20 & 40 \\
\hline Trauma & 342 & 16 & 32 \\
\hline $\begin{array}{l}\text { Reported foreign body (in nose, swallowed, } \\
\text { in eye) }\end{array}$ & 28 & 1 & 3 \\
\hline Poisoning/overdose reported & 19 & 1 & 2 \\
\hline $\begin{array}{l}\text { Musculoskeletal - not trauma (irritable hip, } \\
\text { non-weight bearing, limb aches and pains } \\
\text { not due to trauma) }\end{array}$ & 44 & 2 & 4 \\
\hline Skin & 393 & 18 & 36 \\
\hline Presenting symptom 'Rash' & 233 & 11 & 22 \\
\hline Gastroenterological & 249 & 11 & 23 \\
\hline Vomiting & 111 & 5 & 10 \\
\hline Diarrhoea and vomiting & 81 & 4 & 8 \\
\hline $\begin{array}{l}\text { Non-specific symptoms (crying, floppy, presenting } \\
\text { symptom described as 'fever', generally unwell, } \\
\text { poor intake) }\end{array}$ & 189 & 9 & 18 \\
\hline $\begin{array}{l}\text { Eye presenting symptoms (conjunctivitis, } \\
\text { painful eyes, swollen eyes) }\end{array}$ & 126 & 6 & 12 \\
\hline Genitourinary problems & 40 & 2 & 4 \\
\hline Neurological (febrile convulsions and fitting) & 21 & 1 & 2 \\
\hline \multicolumn{4}{|l|}{ Other } \\
\hline $\begin{array}{l}\text { Includes mumps, meningitis concerns, } \\
\text { symptoms from more than one type, anaemia, } \\
\text { palpitations, teething }\end{array}$ & 69 & 3 & 6 \\
\hline
\end{tabular}

range of primary care providers, were respiratory, followed by trauma, skin and gastroenterological symptoms. When the results were categorised into site of presentation, trauma was the most common at the Emergency Department with respiratory as frequent as trauma and skin presenting symptoms at the WIC and the most common presenting symptom for OoHs and GP surgeries. An interesting finding was that gastroenterological symptoms appeared to present most frequently to the Emergency Department while skin symptoms were seen most commonly at the WIC. Parent-reported presenting symptoms over time showed there was seasonal variation for respiratory, gastroenterological and skin parentreported presenting symptoms. There was a decline for each of the parent-reported presenting symptoms over time. This could be explained by regression to the mean but the numbers for each parent-reported presenting complaint were too small to be able to allow further analysis.

\section{Strengths}

To our knowledge, this is the first study to describe parent-reported presenting symptoms across a range of primary care services since the introduction of WICs and the change in GP OoHs providers. We believe it is also the first study of parent-reported presenting symptoms in preschool children in a UK primary care setting at the time of presentation. A further strength of our study is that we coded parentreported symptoms verbatim, thereby avoiding the use of clinicians' inconsistent diagnoses.

\section{Limitations}

We are aware of three study limitations. First, the main purpose of the PITCH invitation letter 


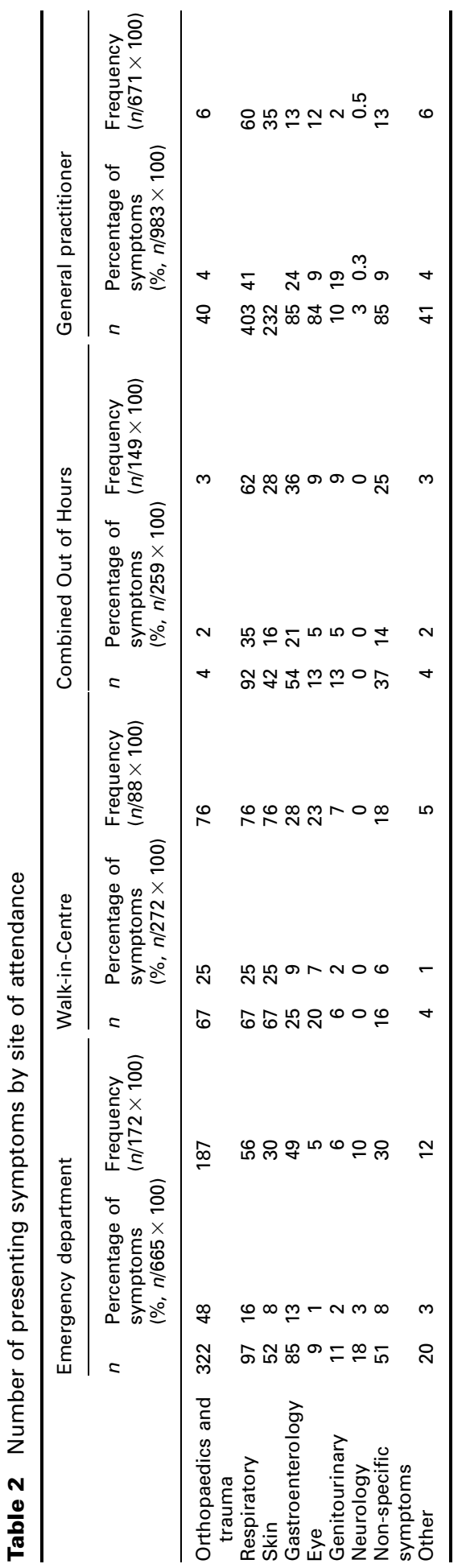

was to identify children with fever to enter the PITCH study. Recording presenting symptoms was a secondary data collection objective and analysed retrospectively. Nearly one third of children's presentations were excluded as there was no record of a parent-reported symptom. This occurred more often for illness presentations with parents responding positively to the fever question. The non-response may have led to an underestimation of the symptom frequencies, and possibly more so for infections, which are associated with fever (eg, respiratory, gastroenterological and genitourinary symptoms). Fever may also be associated with more severe infections and so the results may underestimate more severe illnesses. Some further slight underestimation of symptoms may have occurred due to coding some 'dual symptoms'.

Second, we do not know, and are unable to measure, the between parent reliability of the presenting symptom terms used. Some of the parentreported symptoms codes are very similar in description, for example, chesty, wheezy and chest infection. There are also some codes that could be described as clinical diagnoses rather than presenting symptoms. This may have reflected some health training or knowledge by the parents. Another hypothesis is that the child had already been seen by health professionals earlier in the illness and a diagnosis had been discussed. If the parents then attended again and were logged as a new child illness episode presentation, parents may have described the diagnosis already given instead of the original presenting symptom. As the data were analysed after collection was completed, this could not be further clarified with parents/carers. The use of the parent-reported presenting symptoms verbatim allowed us to explore the type of parentreported presenting symptom in a unique way, but the wide variation and the lack of clarification of parental meaning may have impacted onto the validity and reliability of some results. Future studies with a study design using parental-reported presenting symptoms verbatim as well as a standardised presenting symptom code such as the World Organisation of Family Doctors' International Classification for Primary Care (ICPC-2) may improve reliability (ICPC-2, 1998).

Finally, although comparisons between sites can be made since we report the results per 100 research nurse sessions, recruitment density was higher in the GP practices compared with the other sites.

Primary Health Care Research \& Development 2011; 12: 123-134 


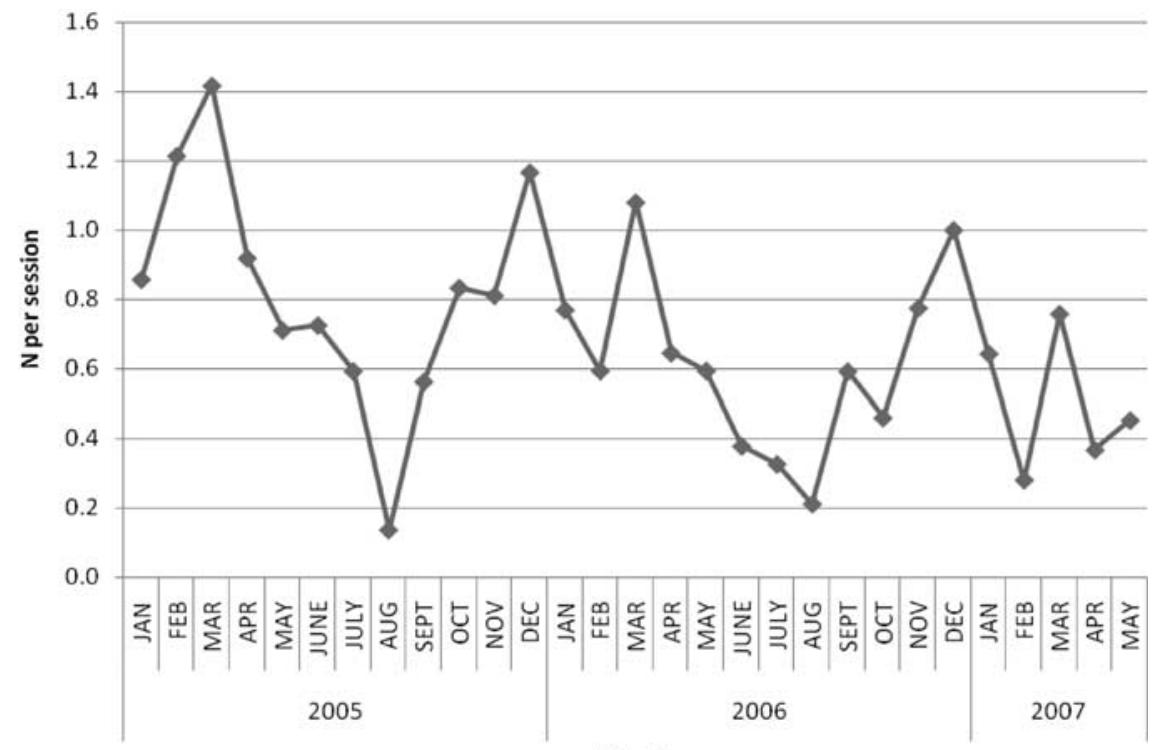

Month

Figure 2 Frequency of respiratory presenting symptoms per session over two-year study period

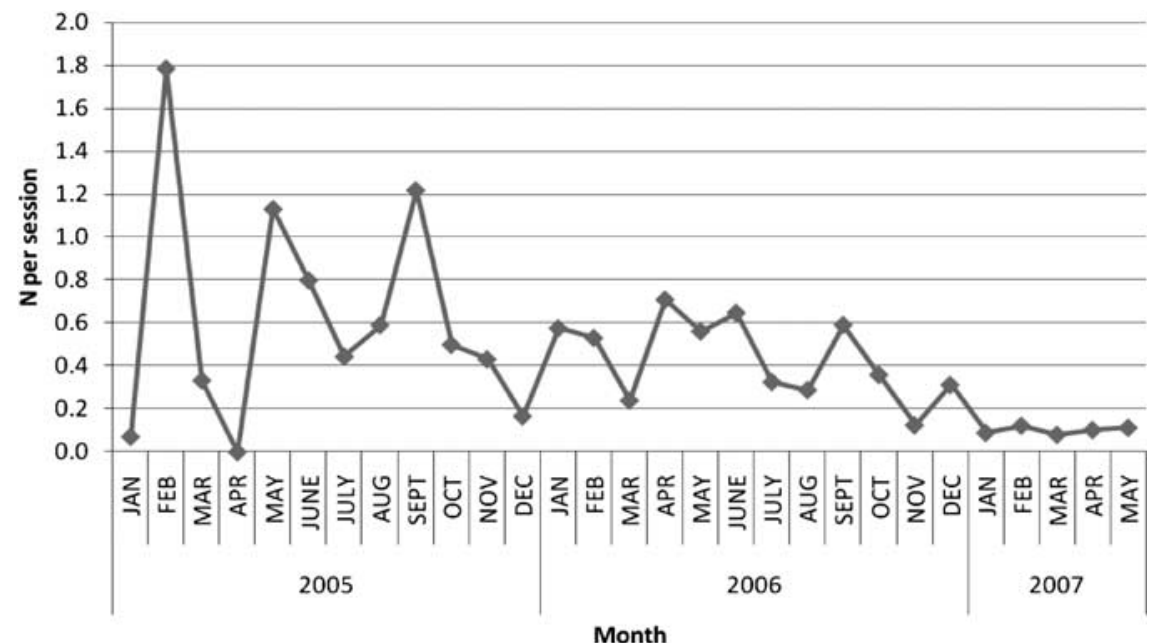

Figure 3 Frequency of trauma presenting symptoms per session over two-year study period

\section{Comparison with other studies}

\section{Presenting symptoms}

Our study found that parents used a wide range of words and phrases to describe the presenting symptom. Other studies have found considerable variability in how parents characterise symptoms (Young et al., 2002). However, when grouped into types, our frequency distribution of parentreported presenting symptoms are similar to the findings of other studies examining symptom incidence and presentation in primary care

Primary Health Care Research \& Development 2011; 12: 123-134 


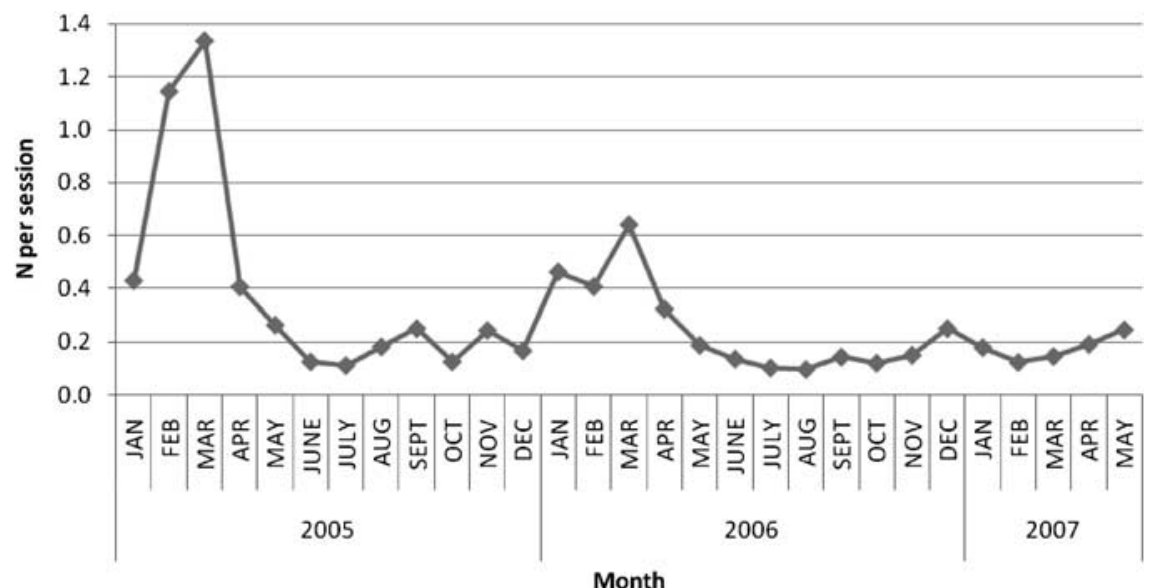

Figure 4 Frequency of gastroenterological presenting symptoms per session over two-year study period

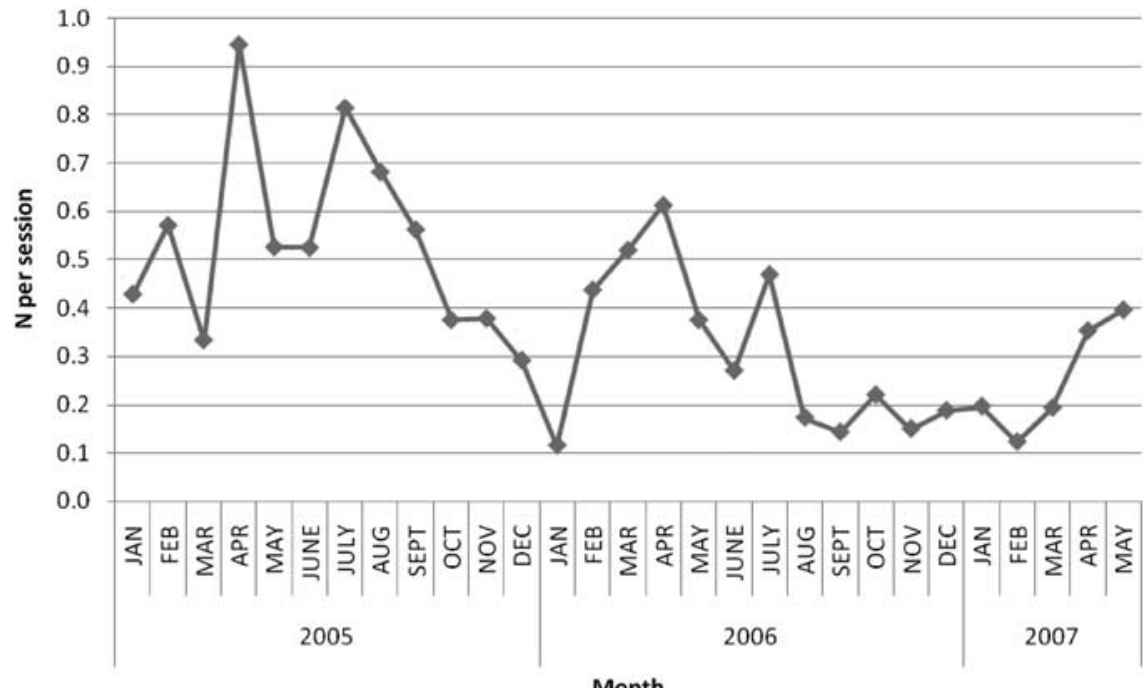

Figure 5 Frequency of skin presenting symptoms per session over two-year study period

(Holme, 1995; Bruijnzeels et al., 1998; Hay et al., 2005) and studies conducted exclusively in Emergency Departments (Phillips and Robson, 1992; Hendry et al., 2005). Our finding that respiratory symptoms were the most frequent presenting symptom except at the Emergency Department agrees with their findings.

One previous study looked at attendances with skin problems at a WIC for ages 2-80 years old (Ersser et al., 2005). In 12 weeks, $21 \%$ of all attendees had a skin-related problem. We found that a similar $25 \%$ of presentations at the WIC had a parentreported skin presenting symptom though in a much narrower age group over a longer period of time.

A Dutch study of 301 children and adults retrospectively compared the records of patients attending an OoHs GP cooperative and an Emergency Department. They found similar results to our study in that the OoHs service dealt with more infectious symptoms than the Emergency 
Department where the most frequent presentation was for trauma (Giesen et al., 2006). However, unlike our study, the authors found that gastroenterological symptoms were only a small percentage of presenting symptoms. This difference may be due to the difference in age groups studied.

\section{Parent-reported symptoms over time}

The seasonal pattern of consultations for children and adolescents with GPs has been observed before (Pace et al., 2004). Our study found similar patterns for respiratory and gastroenterological presenting symptoms. One reason for the seasonality of gastroenterological presenting symptoms may be due to the known seasonality of Rotavirus infections which has been found to be peak in February and March in the preschool age group (Djuretic et al., 1999).

Studies into seasons, weather and frequency of attendances at Emergency Departments have found conflicting outcomes (Attia and Edward, 1998; Rusticucci et al., 2002; Macgregor, 2003). A study in an Argentine Emergency Department looked at a wide range of symptoms including trauma and found a general increase in all types of problem in their winter except for 'genitourinary and abdominal problems' and 'skin and allergies problems' that had more attendances in their summer (Rusticucci et al., 2002).

\section{Implications}

The results of our study show the epidemiology of parent-reported presenting symptoms for a wide range of parent-reported symptom types and primary care services with time. These results could be used by researchers planning recruitment to paediatric primary care studies. The findings can be used to estimate how many children's presentations might be expected for the presenting symptom of their research. This could be used to estimate the time needed for recruitment as well as the recruitment method. For example, a study to test the effectiveness of a new treatment for ottorhoea in children would be ill advised to station research staff in waiting rooms as we did for the PITCH study (Only $0.2 \%$ of illness episodes were parent-reported as 'runny or discharging ear'). The results could also be used by primary care service providers planning healthcare provision and staff training.

\section{Further research}

Two unexpected findings in our study were the frequency of gastroenterological symptoms in the Emergency Department and the number and type of symptoms presenting to the WIC. We know that parents' choice of utilisation is determined by the availability of care, socio-economic factors (Shipman et al., 1997) and their own health beliefs (Hendry et al., 2005). Qualitative research is needed to improve our understanding of parental decision making when choosing which primary care service provider to attend for their children's presenting symptom. Further research into parental meanings and definitions of presenting symptoms would help to increase understanding of parental concerns and reasons for consultation.

The most frequent gastroenterological symptom was vomiting and/or diarrhoea. Parents may attend Emergency Departments due to unknown fears about vomiting and diarrhoea and its acute onset means that the illness may start when parents are unable to access GP services for reassurance. Studies have been done into parents' concerns about fever and wheeze. To improve parent and clinician communication around vomiting and diarrhoea further research into parents' views and concerns about gastroenterological problems may be needed. Further research is also needed into the reasons behind the symptoms presenting to the WIC. There was only one Walk-in Centre in this study and for future service delivery, further research into children's presentation is needed.

Although it is established that many symptoms presentations vary with season, the causative factors have not been identified and should be the subject of further research.

\section{Conclusions}

In conclusion, we found that the most common type of parent-reported presenting symptoms for children aged between six months and six years are respiratory symptoms. When data are grouped by primary care presenting sites, parent-reported respiratory symptoms were the most frequent except for the Emergency Department in which orthopaedic and trauma presenting symptoms were the most common. The estimates of relative symptom presentation frequencies given may help researchers estimating recruitment rates to 
paediatric primary care studies and allow primary care service providers to plan service provision.

\section{Acknowledgements}

This work was supported by the National Institute for Health Research Health Technology Assessment programme (project no. 03/09/01). The final study design, data collection and analysis, interpretation of results and paper writing was the sole responsibility of the authors. For the duration of the trial, $\mathrm{ADH}$ held a postdoctoral award from the National Coordinating Centre for Research Capacity Development, Department of Health. The views and opinions expressed in this paper do not necessarily reflect those of the funding bodies. This study was approved by the Bath research ethics committee (reference no. 04/Q2001/197). The authors declare no competing interests. The authors thank Avon, Gloucestershire and Wiltshire NHS Direct; the Bristol General Practitioner practices; the South Bristol Walk-in-Centre; the Emergency Department of the Bristol Royal Hospital for Children; the children and parents who participated; the South West Medicines for Children Local Research Network; the research nurse team W. Horseman, J. Farrimond, R. Powell, S. Shatford, P. Richards; the South West Medicines for Children Local Research Network nurse V. Payne; W. Patterson (trial coordinator); S. Bryant and S. Burke (project administrators); K. Schroeder, M. Weiss and A. Emond (co-applicants); K. Pitcher (data entry and quality); the trial steering committee (A. L. Kinmonth, C. Butler, J. Peacock, M. Blythe and P. Denyer); and the data monitoring and safety committee (R. Bragonier, S. Kerry and J. Chudleigh). ADH, AAM, MF and TJP conceived the original trial and wrote the protocol. ADH and SW conceived this observational study. The research nurse team collected the data under the supervision of NMR, CC and SH. SW, ADH and AAM cleaned and analysed the data. SW initially drafted the paper, with subsequent contributions from all authors. SW is the guarantor.

\section{References}

Attia, M.W. and Edward, R. 1998: Effect of weather on the number and the nature of visits to a pediatric ED. American Journal of Emergency Medicine 16, 374-75.
Bruijnzeels, M.A., Foets, M., van der Wouden, J.C., van den Heuvel, W.J. and Prins, A. 1998: Everyday symptoms in childhood: occurrence and general practitioner consultation rates. British Journal of General Practice 48, 880-84.

Department of Health. 2000: The NHS Plan: a plan for investment, a plan for reform. London: The Stationery Office, Cm 4818-I.

Djuretic, T., Ramsay, M., Gay, N., Wall, P., Ryan, M. and Fleming, D. 1999: An estimate of the proportion of diarrhoeal disease episodes seen by general practitioners attributable to rotavirus in children under $5 \mathrm{y}$ of age in England and Wales. Acta Paediatrica Supplement 88, 38-41.

Ersser, S.J., Lattimer, V., Surridge, H. and Brooke, S. 2005: An analysis of the skin care patient mix attending a primary care-based nurse-led NHS Walk-in Centre. British Journal of Dermatology 153, 992-96.

Giesen, P., Franssen, E., Mokkink, H., van den, B.W., van, V.A. and Grol, R. 2006: Patients either contacting a general practice cooperative or accident and emergency department out of hours: a comparison. Emergency Medical Journal 23, 731-34.

Hay, A.D., Heron, J. and Ness, A. 2005: The prevalence of symptoms and consultations in pre-school children in the Avon Longitudinal Study of Parents and Children (ALSPAC): a prospective cohort study. Family Practice 22, 367-74.

Hay, A.D., Costelloe, C., Redmond, N.M., Montgomery, A.A., Fletcher, M., Hollinghurst, S. and Peters, T.J. 2008: Paracetamol plus ibuprofen for the treatment of fever in children (PITCH): randomised controlled trial. British Medical Journal 337, a1302-10.

Hendry, S.J., Beattie, T.F. and Heaney, D. 2005: Minor illness and injury: factors influencing attendance at a paediatric accident and emergency department. Archives of Disease in Childhood 90, 629-33.

Holme, C.O. 1995: Incidence and prevalence of non-specific symptoms and behavioural changes in infants under the age of two years. British Journal of General Practice 45, 65-69.

ICPC-2. 1998: International classification of primary care, second edition. Prepared by the International Classification Committee of WONCA. Oxford: Oxford University Press.

Kai, J. 1996: What worries parents when their preschool children are acutely ill, and why: a qualitative study. British Medical Journal 313, 983-86.

Little, P., Somerville, J., Williamson, I., Warner, G., Moore, M., Wiles, R., George, S., Smith, A. and Peveler, R. 2001: Family influences in a cross-sectional survey of higher child attendance. British Journal of General Practice. 51, 977-81, 984.

Macgregor, D.M. 2003: Effect of weather on attendance with injury at a paediatric emergency department. Emergency Medical Journal 20, 204-205.

McCormick, A., Fleming, D. and Charlton, J. 1995: Morbidity statistics from general practice. Fourth National study 1991-1992. London: HMSO. 


\section{Sara Whitburn et al.}

Medical Research Council. 1997: MRC topic review, primary health care research review. London: Medical Research Council.

Morrell, D.C. 1970: Presenting symptoms in general practice. British Journal of Preventive and Social Medicine 24, 64.

Osman, L.M. and Dunt, D. 1995: Factors influencing mothers' decisions to consult a general practitioner about their children's illnesses. British Journal of General Practice 45, 310-12.

Pace, W.D., Dickinson, L.M. and Staton, E.W. 2004: Seasonal variation in diagnoses and visits to family physicians. Annals of Family Medicine 2, 411-17.

Pattison, C.J., Drinkwater, C.K. and Downham, M.A. 1982: Mothers' appreciation of their children's symptoms. The Journal of the Royal College of General Practice 32, 149-62.

Phillips, B.M. and Robson, W.J. 1992: Paediatrics in the accident and emergency department. Archive of Disease in Childhood 67, 560-64.

Robertson-Steel, I.R. 1998: Providing primary care in the accident and emergency department. British Medical Journal 316, 409-10.
Rowlands, S. and Moser, K. 2002: Consultation rates from the general practice research database. British Journal of General Practice 52, 658-60.

Rusticucci, M., Bettolli, M.L. and de los Angeles Harris, M. 2002: Association between weather conditions and the number of patients at the emergency room in an Argentine hospital. International Journal of Biometeorology 46, $42-51$.

Shipman, C., Longhurst, S., Hollenbach, F. and Dale, J. 1997: Using out-of-hours services: general practice or A\&E? Family Practice 14, 503-509.

Stocks, N. and Fahey, T. 2002: Labelling of acute respiratory illness: evidence of between-practitioner variation in the UK. Family Practice 19, 375-77.

Wyke, S., Hewison, J. and Russell, I.T. 1990: Respiratory illness in children: what makes parents decide to consult? British Journal of General Practice 40, 226-29.

Young, B., Fitch, G.E., Dixon-Woods, M., Lambert, P.C. and Brooke, A.M. 2002: Parents' accounts of wheeze and asthma related symptoms: a qualitative study. Archive of Disease in Childhood 87, 131-34. 